\title{
Feuer frei auf das Wolfsrudel?
}

\author{
- §45a Abs. 2 BNatSchG im Lichte der FFH-Richtlinie \\ Anne-Christin Gläß und Alexander Brade
}

\begin{abstract}
(C) Der/die Autor(en) 2021. Dieser Artikel ist eine Open-Access-Publikation.
Der vorliegende Beitrag widmet sich dem Zweiten Gesetz zur Änderung des Bundesnaturschutzgesetzes (BNatSchG), das am 13. 3. 2020 in Kraft getreten ist. Dabei gibt es berechtigte Zweifel, ob es sein eigentliches Ziel erreichen wird: durch eine bundeseinheitliche Regelung - einzelnen landesspezifischen Rechtsverordnungen nachfolgend - Rechtssicherheit für die Erteilung artenschutzrechtlicher Ausnahmegenehmigungen zu schaffen und so den Vollzug zu erleichtern. Die Änderungen, allen voran der neu ins Gesetz aufgenommene $\int 45$ a Abs. 2 BNatSch G, werfen die Frage nach der Vereinbarkeit mit dem Unionsrecht (neu) auf. Dies gilt besonders im Lichte der jüngsten Rechtsprechung des EuGH, der mit Urteilen vom 10. 10.2019 und 11. 6. 2020 nochmals den hohen Wert des Artenschutzes und die daraus folgenden Verpflichtungen für den Umgang mit dem Wolf hervorgehoben hat.
\end{abstract}

\section{Einleitung}

Der Wolf ist spätestens seit seiner Rückkehr nach Deutschland ${ }^{1}$ in aller Munde; gerungen wird um den Schutz des Wolfes einerseits und die Sicherheit des Menschen und seiner Nutztiere andererseits. ${ }^{2}$ Daraus erwächst auch eine zunehmende Anzahl juristischer Konflikte: Die juris-Datenbank listet allein für den Zeitraum 2019 bis Juni 2020 eine Reihe verwaltungsgerichtlicher Entscheidungen auf, die sich mit Rechtsfragen zum Umgang mit dem Wolf befassen. ${ }^{3}$ Mit der neuen Vorschrift des $\$ 45 \mathrm{a} B N a t S c h G^{4}$ hatte sich dagegen jüngst erstmals das VG Lüneburg und - auf die Beschwerde gegen diese Entscheidung hin - das OVG Lüneburg zu befassen. Bereits das VG äußerte rechtliche Bedenken gegen den Bescheid, da die schadensverursachenden Wölfe feststünden und der Schaden nicht, wie $\$ 45$ a Abs. 2 BNatSchG es verlange, keinem bestimmten Wolf eines Rudels zugeordnet werden könnte. ${ }^{5}$ Das OVG tendierte nun zwar zu einer erweiternden Auslegung, nach der auch Fälle erfasst sein sollten, in denen die gezielte Tötung des konkreten Wolfs in der Natur mangels klarer Erkennbarkeit schwierig durchzuführen ist. ${ }^{6}$ Das OVG ließ diese Frage aber letztlich dahinstehen, da die Voraussetzungen des $\ 45$ a Abs. 2 S. 1 BNatSchG jedenfalls nicht erfüllt seien. ${ }^{7}$ Eine Aussage zur Unionsrechtskonformität der Norm ist damit, soweit ersichtlich, nicht verbunden ${ }^{8}$ und wäre auch mehr als überraschend, da es mangels Entscheidungserheblichkeit an der Erforderlichkeit einer Vorabentscheidung i.S.d. Art. 267 AEUV gefehlt haben dürfte. Und dennoch: Es mehren sich die Anzeichen dafür, dass sich der EuGH mit dieser, auch hier im Mittelpunkt stehenden Frage wird auseinandersetzen müssen, sei es im Wege des Vorabentscheidungs- oder des Vertragsverletzungsverfahren. Allerdings ist die Problematik - das sei am Rande angemerkt - darauf bekanntlich nicht beschränkt: Zwar spricht die Ausnahmeregelung des $₫ 45$ Abs. 7 S. 1 Nr. 1 BNatSchG nunmehr wie Art. 16 Abs. 1 lit. b) FFH-Richtlinie ${ }^{9}$ von „,ernsten“ Schäden, erstreckt sich dabei aber auch auf, ,sonstige (ernste) wirtschaftliche Schäden“. Das mag sich bei richtlinienkonformer Aus-

Dr. Anne-Christin Gläß, LL.M.Eur, wiss. Mitarbeiterin und Dr. Alexander Brade, wiss. Mitarbeiter,

beide am Lehrstuhl für Öffentliches Recht bei

Prof. Dr. Kurt Faßbender, insbesondere Umwelt- und Planungsrecht, Universität Leipzig, Leipzig, Deutschland legung noch in dem von der FFH-Richtlinie vorgegebenen Rahmen bewegen, ${ }^{10}$ weicht aber wie schon die Vorgängerregelung zu $\$ 45$ Abs. 7 S. 1 Nr. 1 BNatSchG von Art. 9 Abs. 1 lit. a) Vogelschutz-Richtlinie ${ }^{11} \mathrm{ab}$, der den Schutz ,sonstiger Formen des Eigentums“" nach seinem insoweit abschließend $\mathrm{zu}$ verstehenden Normtext ${ }^{12}$ nicht anerkennt. ${ }^{13}$

1) Detaillierte und aktuelle Informationen zum Wolfsvorkommen in Deutschland bietet die Dokumentations- und Beratungsstelle des Bundes zum Thema Wolf, Stand 14.7.2020, abrufbar unter https://www.dbb-wolf.de/Wolfsvorkommen. Zum tatsächlichen Status des Wolfes auch Köck/Kuchta, NuR 2017, 509, $511 \mathrm{f}$.

2) Vgl. nur Köck, NuR 2018, 812f.; Rüwe, NdsVBl. 2020, 65

3) S. etwa VG Oldenburg, Beschl. v. 15.2.2019 - 5 B 472/19, NuR 2019, 287; OVG Lüneburg, Beschl. v. 22.2.2019 - 4 ME 48/19, NuR 2019, 272; VG Gera, Beschl. v. 20.2.2020 - 5 E 67/20 Ge, ZUR 2020, 313 (jeweils zu Ausnahmegenehmigungen für die Tötung eines Wolfs); OVG Bautzen, Urt. v. 25.2.2020 - 4 A 930/17, juris (Schadensausgleich wegen der Tötung mehrerer Pferde durch einen Wolf).

4) Gesetz über Naturschutz und Landschaftspflege (Bundesnaturschutzgesetz - BNatSchG) v. 29.7.2009, BGBl. I S. 2542; zuletzt geändert durch Art. 1 Zweites ÄndG v. 4.3.2020, BGBl. I S. 440.

5) VG Lüneburg, Beschl. v. 18.5.2020 - 2 B 31/20, 2 B 34/20, juris

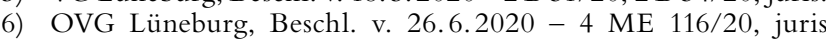
Rdnr. 45.

7) OVG Lüneburg, Beschl. v. 26.6.2020 - 4 ME 116/20, juris Rdnr. $45 \mathrm{ff}$

8) OVG Lüneburg, Beschl. v. 26.6.2020 - 4 ME 116/20, juris Rdnr. 47, weist lediglich darauf hin, dass auch die Auslegung des $₫ 45$ a Abs. 2 S. 1 den europarechtlichen Anforderungen genügen müsse.

9) Richtlinie 92/43/EWG des Rates vom 21.5.1992 zur Erhaltung der natürlichen Lebensräume sowie der wildlebenden Tiere und Pflanzen, ABl. 1992 L 206, S. 7 (zuletzt geändert durch Art. 1 ÄndRL 2013/17/EU vom 13. 5. 2013, ABl. 2013 L 158, S. 193).

10) Näher Gläß, in: Giesberts/Reinhardt (Hrsg.), BeckOK, Umweltrecht, 54. EL (4/2020), \45 BNatSchG Rdnr. 39. A. A. Borwieck, ZUR 2020, 50, 52 zur Ursprungsfassung der Neuregelung, die nur von ,sonstigen ernsten Schäden“ sprach. Zum Teil wird davon ausgegangen, dass der Schaden im Zusammenhang mit beruflicher Tätigkeit bzw. gewerblich genutztem Eigentum stehen muss, sodass Schäden bei der Hobbytierhaltung, die nunmehr von $\ 45$ a Abs. 2 S. 2 BNatSchG geregelt wird, nicht erfasst würden (Kremer, Stellungnahme zum Entwurf eines Zweiten Gesetzes zur Änderung des BNatSchG, Ausschussdrucksache 19(16)308-D, S. 3; a. A. Jessel, Stellungnahme zum Entwurf eines Zweiten Gesetzes zur Änderung des BNatSchG, Ausschussdrucksache 19(16)308-F, S. 2f.; Rüwe, NdsVBl. 2020, 65, 69).

11) Richtlinie 2009/147/EG des Europäischen Parlaments und des Rates vom 30.11.2009 über die Erhaltung der wildlebenden Vogelarten, AB1. 2010 L 20, S. 7 (zuletzt geändert durch Art. 5 VO (EU) 2019/1010 vom 5.6.2019, ABl. 2019 L 170, S. 115.)

12) Vgl. nur EuGH, Urt. v. 12.7.2007 - C-507/04, NuR 2007, 537, Rdnr. 326.

13) Gellermann, Stellungnahme zum Entwurf eines Zweiten Gesetzes zur Änderung des BNatSchG, Ausschussdrucksache 19(16)308-C, S. 2f. und Kremer, Stellungnahme zum Entwurf eines Zweiten Gesetzes zur Änderung des BNatSchG, Ausschussdrucksache 19(16)308-D, S. 3 halten die Regelung daher für mit der Vogelschutz-Richtlinie nicht vereinbar und unionsrechtswidrig. Näher Gläß, in: Giesberts/Reinhardt (Hrsg.), BeckOK-Umweltrecht, 54. EL (4/2020), \45 BNatSchG Rdnr. 49 u. 51 m. w. N. und dem Hinweis darauf, dass vor dem Hintergrund zunehmender Zweifel in Literatur und Rechtsprechung im Interesse der Rechtssicherheit jedenfalls eine Klärung durch den EuGH wünschenswert erscheine. 


\section{Inhalt der deutschen Sonderregelung zur Entnahme von Wölfen}

Nach der allgemeinen Ausnahmevorschrift des $₫ 45$ Abs. 7 S. 1 Nr. 1 BNatSchG kann die zuständige Naturschutzbehörde von den Verboten des $\$ 44-$ damit auch vom Tötungsverbot des $\ 44$ Abs. $1 \mathrm{Nr} .1 \mathrm{BNatSchG}$ - im Einzelfall Ausnahmen zur Abwendung ernster land-, forst-, fischerei- oder wasserwirtschaftlicher oder sonstiger ernster wirtschaftlicher Schäden zulassen. Der neue $\$ 45$ a Abs. 2 BNatSchG nimmt unmittelbar Bezug auf eben diese Norm und regelt Besonderheiten bei der Anwendung der Bestimmung auf die (letale) Entnahme von Wölfen. \45a Abs. 2 BNatSchG gestaltet die Möglichkeit der zuständigen Behörden zum Erlass von Ausnahmen näher aus, nimmt sie aber noch nicht vorweg; die Genehmigung einer konkreten Ausnahme bleibt weiterhin als Einzelfallentscheidung der jeweils zuständigen Behörde überlassen. Dabei stellt diese Norm in zweierlei Hinsicht eine Sonderregelung dar: zum einen mit Blick auf den abzuwendenden Schaden (Satz 2 der Vorschrift), zum anderen hinsichtlich der grundlegenden Anforderungen an den Nachweis der Geeignetheit einer Entnahme von Wölfen zur Abwehr des (drohenden) Schadens (Satz 1 der Vorschrift).

\subsection{Regelung zum abzuwendenden Schaden}

$\$ 45$ a Abs. 2 S. 2 BNatSchG befasst sich mit den ,ernsten wirtschaftlichen Schäden“, die eine Ausnahmeentscheidung rechtfertigen können. Ziel der Gesetzesänderung war es insofern, auch Hobbyhalter von Weidetieren vor Rissereignissen durch den Wolf zu schützen $;{ }^{14}$ auch wenn nicht landwirtschaftlich gehaltene Weidetiere gerissen werden, sollen nun ernste wirtschaftliche Schäden drohen können.

Dabei geht es der neuen Regelung allerdings nicht lediglich um die als Hobby - statt zur Gewinnerzielung - betriebene Weidetierhaltung als solche. Zu beachten ist, dass das Kriterium des ernsten wirtschaftlichen Schadens keineswegs aufgegeben wird und vor dem europarechtlichen Hintergrund ${ }^{15}$ auch nicht ohne weiteres aufgegeben werden dürfte. Der (drohende) Schaden, der mittels der Entnahmeentscheidung abgewendet werden soll, muss auch weiterhin eine gewisse Erheblichkeitsschwelle überschreiten. ${ }^{16}$ Von einer "Aufweichung“ der Anforderung in $\$ 45$ Abs. 7 S. 1 Nr. 1 BNatSchG selbst wurde im Verlauf des Gesetzgebungsverfahrens gerade Abstand genommen. ${ }^{17}$

Die Einbeziehung auch der Hobbytierhalter in den besonderen Schutz, den $\$ 45 \mathrm{a}$ Abs. 2 BNatSchG im Umgang mit dem Wolf gewährleisten soll, wird damit gerechtfertigt, dass nach einmaligem Überwinden von Herdenschutzmaßnahmen - sei es auch bei denjenigen eines Hobbytierhalters - auch künftig mit dem Überwinden solcher Maßnahmen durch das jeweilige Tier zu rechnen ist. Der Wolf wird nicht zwischen Hobbytierhaltern und landwirtschaftlichen Betrieben unterscheiden, hat er einmal Herdenschutzmaßnahmen überwunden. ${ }^{18}$ Vom bereits eingetretenen Schaden eines Hobbytierhalters schließt der Gesetzgeber damit auf den künftig zumindest drohenden Schaden auch für landwirtschaftliche Betriebe in der betreffenden Region. Dieser Schluss darf aber nicht dazu führen, dass das Kriterium des ernsten bzw. erheblichen Schadens durch eine Art Automatismus preisgegeben wird. Auch im Fall eines eingetretenen Schadens an nicht landwirtschaftlich gehaltenen Weidetieren ist daher - vor dem Hintergrund fachlicher Schlussfolgerungen aufgrund der überwundenen Schutzvorrichtungen mit Blick auf die konkreten Gegebenheiten darzulegen, dass künftig ein ernster wirtschaftlicher Schaden zumindest droht.

Eine besondere Voraussetzung für den Fall, dass aufgrund von Rissereignissen bei lediglich hobbymäßig gehaltenen Weidetieren eine Entnahme von Wölfen genehmigt werden soll, nennt $\int 45 a$ Abs. 2 S. 2 BNatSchG zusätzlich: Die nicht landwirtschaftlich gehaltenen Weidetiere müssen durch zumutbare Herdenschutzmaßnahmen geschützt gewesen sein. Ungeachtet dieser ausdrücklichen Regelung in $₫ 45 \mathrm{a}$ Abs. 2 S. 2 BNatSchG sind derartige Herdenschutzmaßnahmen ${ }^{19}$ allerdings auch in allen anderen Fällen vorrangig heranzuziehen. Dies verlangt bereits das Erfordernis der Alternativenprüfung in $\ 45$ Abs. 7 S. 2 BNat$\mathrm{SchG}^{20}$ : Sind zumutbare Alternativen, etwa in Gestalt von Herdenschutzmaßnahmen möglich, so ist die Entnahme streng geschützter Wölfe nicht erforderlich und nach den Bestimmungen des europäischen und nationalen Artenschutzrechts unzulässig. Nicht zuletzt die finanzielle Förderung derartiger Herdenschutzmaßnahmen macht diese im Übrigen auch grundsätzlich zumutbar. ${ }^{21}$

\subsection{Nachweis der Geeignetheit einer konkreten Entnahmeentscheidung}

Als von noch größerer Bedeutung für die Praxis könnte sich allerdings $₫ 45 \mathrm{a}$ Abs. $2 \mathrm{~S} .1 \mathrm{BNatSchG}$ erweisen. Die eine Ausnahmegenehmigung rechtfertigenden Schäden i.S.d. $\$ 45$ Abs. 7 S. 1 Nr. 1 BNatSchG müssen grundsätzlich konkret dem Tier zugeordnet werden, für das eine Entnahmegenehmigung erteilt wird. Allerdings befreit $\$ 45 \mathrm{a}$ Abs. 2 S. 1 BNatSchG die zuständige Behörde nunmehr unter bestimmten Voraussetzungen von diesem in der Praxis zum Teil schwer zu leistenden Erfordernis der konkreten $\mathrm{Zu}$ ordnung. Voraussetzung dafür ist zunächst, dass Schäden in Form von Nutztierrissen vorliegen, die keinem bestimmten Wolf eines Rudels zugeordnet werden können. ${ }^{22}$ Dies soll

14) Vgl. die Begründung des Gesetzentwurfs der Bundesregierung, BT-Drs. 19/10899, S. 9, sowie - darauf Bezug nehmend - die Beschlussempfehlung und Bericht des Ausschusses für Umwelt, Naturschutz und nukleare Sicherheit, BT-Drs. 19/16148, S. 9.

15) Dazu sogleich unter 3.1 und 3.2.

16) Vgl. allgemein zur Voraussetzung des $₫ 45$ Abs. 7 S. 1 Nr. 1: Gläß, in: Giesberts/Reinhardt (Hrsg.), BeckOK-Umweltrecht, 54. EL (4/2020), §45 BNatSchG Rdnr. 39 ff.; noch zur früheren Gesetzesfassung Lau, in: Frenz/Müggenborg, BNatSchG, 2. Aufl. 2016, \45 Rdnr. 14; Lütkes, in: Lütkes/Ewer, BNatSchG, 2. Aufl. 2018, ऽ 45 Rdnr. $30 \mathrm{f}$.

17) Siehe die Beschlussempfehlung und Bericht des Ausschusses für Umwelt, Naturschutz und nukleare Sicherheit, BT-Drs. 19/16148, S. 9.

18) Vgl. zu diesem Hintergrund der Gesetzesänderung Beschlussempfehlung und Bericht des Ausschusses für Umwelt, Naturschutz und nukleare Sicherheit, BT-Drs. 19/16148, S. 9.

19) Einen Überblick über derartige Herdenschutzmaßnahmen bietet Bundesamt für Naturschutz (Hrsg.), Empfehlungen zum Schutz von Weidetieren und Gehegewild vor dem Wolf - Konkrete Anforderungen an die empfohlenen Präventionsmaßnahmen, BfNSkripten 530, 2019, Stand 22.6.2020, abrufbar unter https:// www.bfn.de/fileadmin/BfN/service/Dokumente/skripten/ Skript530.pdf. Auch einzelne Länder bieten gezielt Informationen zum Umgang mit dem Wolf an, so beispielsweise auch der Freistaat Sachsen - vgl. etwa zum Herdenschutz die Broschüre des Landesamtes für Umwelt, Landwirtschaft und Geologie (Hrsg.), Herdenschutzhunde und sichere Einzäunung - Hinweise zum Schutz vor dem Wolf, 2018, Stand 22.6.2020, abrufbar unter https://publikationen.sachsen.de/bdb/artikel/22816.

20) Zur Alternativenprüfung Gläß, in: Giesberts/Reinhardt (Hrsg.), BeckOK-Umweltrecht, 54. EL (4/2020), \45 BNatSchG Rdnr. 54; Lau, in: Frenz/Müggenborg, BNatSchG, 2. Aufl. 2016, §45 Rdnr. 21 ff., jeweils m. w. N.

21) Zur finanziellen Förderung - auch für Hobbytierhalter - beispielsweise im Freistaat Sachsen nach der Förderrichtlinie Natürliches Erbe v. 15. 12.2014, SächsABl. SDr. 2015 S. S 28, zuletzt geändert durch die Richtlinie v. 18.3.2020, SächsABl. S. 369, zuletzt enthalten in der Verwaltungsvorschrift v. 9.12.2019, SächsABl. SDr. S. S 414, siehe die näheren Informationen des Sächsischen Staatsministeriums für Energie, Klimaschutz, Umwelt und Landwirtschaft, Stand 22.6.2020, abrufbar unter https://www.smul.sachsen.de/ foerderung/praevention-vor-wolfsschaeden-e-4633.html.

22) So der Wortlaut - zu einer möglichen erweiternden Auslegung s. aber OVG Lüneburg, Beschl. v. 26.6.2020 - 4 ME 116/20, juris Rdnr. 45. 
eine Genehmigung zur Entnahme irgendeines Wolfes des Rudels bereits rechtfertigen können, wenn der Abschuss in engem räumlichem und zeitlichem Zusammenhang mit den bereits eingetretenen Rissereignissen erfolgt.

Kommt es in der Folge auch nach Abschuss eines Wolfes zu weiteren Nutztierrissen, so können unter der eben genannten Voraussetzung zudem weitere entsprechende Ausnahmegenehmigungen erteilt werden - nach Abs. 2 S. 1 bis zum Ausbleiben von Schäden. Auch dies setzt freilich wiederum voraus, dass der Abschuss jeweils in engem räumlichem und zeitlichem Zusammenhang mit den bereits eingetretenen Rissereignissen fortgeführt wird. Im Extremfall könnte auf diese Weise über sukzessive aufeinanderfolgende Entnahmeentscheidungen der Abschuss aller Wölfe eines Rudels genehmigt werden. ${ }^{23}$

$\int 45 a$ Abs. 2 S. 3 BNatSchG erstreckt diese Besonderheiten schließlich auch auf den Fall, dass eine behördliche Entnahmeentscheidung gemäß $\int 45$ Abs. 7 S. 1 Nr. 4 BNatSchG im Interesse der menschlichen Gesundheit erteilt werden soll. Auch in einem solchen Fall soll künftig nicht zwingend die eindeutige Zuordnung der Gefährdung $\mathrm{zu}$ einem bestimmten Wolf eines Rudels erforderlich sein und im Einzelfall gar der Abschuss aller Wölfe eines Rudels erlaubt werden können.

Insbesondere $₫ 45$ a Abs. 2 S. 1 und 3 BNatSchG werfen eine Reihe von Fragen auf. Ob diese Regelung mit den unionsrechtlichen Vorgaben im Artenschutzrecht vereinbar ist, erscheint zumindest zweifelhaft. Dieser Frage soll im folgenden Abschnitt weiter nachgegangen werden.

\section{Vereinbarkeit mit dem Unionsrecht}

\subsection{Der unionsrechtliche Rahmen}

HauptzielderFlora-Fauna-Habitat-Richtlinie(FFH-Richtlinie) ist es, die Erhaltung der biologischen Vielfalt zu fördern, wobei jedoch die wirtschaftlichen, sozialen, kulturellen und regionalen Anforderungen berücksichtigt werden sollen. ${ }^{24} \mathrm{Zu}$ diesem Zweck sieht sie einerseits die Ausweisung besonderer Schutzgebiete vor (Art. $3 \mathrm{ff}$. FFH-Richtlinie) und verpflichtet die Mitgliedstaaten der Union andererseits ein allgemeines Schutzsystem für bestimmte Tiere einzuführen (Art. $12 \mathrm{ff}$. FFH-Richtlinie). Streng geschützt ist danach unter anderem der Wolf (Canis lupus), Art. 12 Abs. 1 i. V.m. Anhang IV lit. a) FFH-Richtlinie; grundsätzlich sind alle absichtlichen Formen des Fangs oder der Tötung von aus der Natur entnommenen Exemplaren dieser Art verboten. Davon können die Mitgliedstaaten nach Art. 16 Abs. 1 FFH-Richtlinie - sofern es erstens keine anderweitige zufriedenstellende Lösung gibt und zweitens unter der Bedingung, dass die Populationen der betroffenen Art in ihrem natürlichen Verbreitungsgebiet trotz der Ausnahmeregelung ohne Beeinträchtigung in einem günstigen Erhaltungszustand ${ }^{25}$ verweilen - zu folgenden Zwecken abweichen:

- Schutz der wildlebenden Tiere und Pflanzen und zur Erhaltung der natürlichen Lebensräume (lit. a);

- Verhütung ernster Schäden insbesondere an Kulturen und in der Tierhaltung sowie an Wäldern, Fischgründen und Gewässern sowie an sonstigen Formen von Eigentum (lit. b);

- Volksgesundheit und öffentliche Sicherheit oder andere zwingende Gründe des überwiegenden öffentlichen Interesses, einschließlich solcher sozialer oder wirtschaftlicher Art oder positiver Folgen für die Umwelt (lit. c);

- Forschung und Unterricht, Bestandsauffüllung und Wiederansiedlung und die für diese Zwecke erforderliche Aufzucht, einschließlich der künstlichen Vermehrung von Pflanzen (lit. d);

- selektive, im Ausmaß beschränkte und streng kontrollierte Entnahme oder Haltung einer begrenzten und von den zuständigen einzelstaatlichen Behörden spezifizierten Anzahl von Exemplaren des Anhangs IV der FFH-Richtlinie (lit. e).

\subsection{Leitentscheidungen des EuGH}

Erstmals mit der praktischen Anwendung dieser Normen gerade auf den Wolf war der EuGH, soweit ersichtlich, mit Urteil vom 14.6.2007 befasst. Dabei versteht er Art. 16 Abs. 1 FFH-Richtlinie - wie schon die Generalanwältin in ihren Schlussanträgen ${ }^{26}$ - als eng auszulegende Ausnahmeregelung, bei der die Beweislast für das Vorliegen der für jede Abweichung erforderlichen Voraussetzungen die Stelle trifft, die über sie entscheidet. ${ }^{27}$ Ein Schaden müsse deshalb zwar nicht abgewartet werden, bevor Maßnahmen ergriffen würden, allerdings verstoße die präventive Tötung von Wölfen dann gegen Art. 12 Abs. 1 und Art. 16 Abs. 1 lit. b) FFH-Richtlinie, wenn es - wie in dem hier zu entscheidenden Fall der Republik Finnland - an einem ausreichenden Nachweis dafür fehle, dass die Jagd zur Verhütung ernster Schäden tatsächlich geeignet ist. ${ }^{28}$ Ohne Einfluss darauf sei der Umstand, dass der Wolf ein Tier ist, das im Allgemeinen im Rudel lebt; zu erbringen sei der (naturwissenschaftliche) Beleg, dass und in welcher Form nicht individualisierte Abschussgenehmigungen dazu beitragen, ernsthafte Schäden zu verhindern..$^{29}$

An seinen strikten Anforderungen an die Geeignetheit des jeweiligen Ausnahmetatbestands hält der EuGH auch in Sachen „Tapiola“, einer Vorabentscheidung erneut die Wolfsjagd in Finnland betreffend, fest. So unterfalle die Bekämpfung der Wilderei als Ziel zwar der (subsidiären) Auffangregelung des Art. 16 Abs. 1 lit. e) FFH-Richtlinie. ${ }^{30}$ Gleichwohl müsse, unabhängig davon, ob sich die Jagd (generell) auf junge Individuen oder solche, die Schäden verursachen, richte, ${ }^{31}$ die Annahme gerechtfertigt sein, dass eine beschränkte Erlaubnis der legalen Jagd zur Eindämmung der Wilderei und damit zur Verbesserung des Erhaltungszustands des Wolfs beitragen könne. ${ }^{32}$ Das bloße Be-

23) So auch ausdrücklich die Begründung des Gesetzentwurfs der Bundesregierung, BT-Drs. 19/10899, S. 10: „bis zur Entnahme des gesamten Rudels".

24) Vgl. Erwägungsgrund Nr. 3 der FFH-Richtlinie.

25) Der Erhaltungszustand von Arten wird in Art. 1 lit. i) FFH-Richtlinie definiert.

26) Generalanwältin Kokott, Schlussanträge v. 30.11.2006 C-342/05, ECLI:EU:C:2006:752, Rdnr. 20 u. 23.

27) EuGH, Urt. v. 14. 6.2007 - C-342/05, NuR 2007, 477, Rdnr. 25

28) EuGH, Urt. v. 14.6.2007-C-342/05, NuR 2007, 477, Rdnr. 40.

29) Generalanwältin Kokott, Schlussanträge v. 30.11.2006 C-342/05, ECLI:EU:C:2006:752, Rdnr. 36; ähnlich EuGH, Urt. v. 14.6.2007 - C-342/05, NuR 2007, 477, Rdnr. $41 \mathrm{ff}$.

30) Vgl. EuGH, Urt. v. 10.10.2019 - C-674/17, NuR 2019, 756, Rdnr. 36 ff.; Klausmann, Mehr Artenschutz durch den EuGH? - Der unionsrechtliche Rahmen zum Schutz von Wolfspopulationen, Stand 29.6.2020, abrufbar unter https://www.juwiss.de/2-2020/beschreibt den Charakter von Art. 16 Abs. 1 lit. e) FFH-Richtlinie als „Ausnahme unter Ausnahmen“.

31) EuGH, Urt. v. 10. 10.2019-C-674/17, NuR 2019, 756, Rdnr. 76

32) EuGH, Urt. v. 10.10.2019-C-674/17, NuR 2019, 756, Rdnr. 44. Nach Auffassung des Generalanwalts könne beim Erlass solcher Ausnahmeregelungen nicht verlangt werden, dass ,ohne jede wissenschaftliche Unsicherheit nachgewiesen ist, dass sie die rechtswidrige Jagd verringern würde und das Ausmaß dieser Wirkung so groß wäre, dass die gesamte dem Menschen zurechenbare Mortalität gesenkt würde." Die nationalen Behörden seien vielmehr unter Zugrundelegung des Vorsorgegrundsatzes nur verpflichtet, ,auf der Grundlage streng wissenschaftlicher Erkenntnisse die Annahme zu untermauern, dass die Genehmigung der bestandspflegenden Jagd die rechtswidrige Jagd verringern würde, und zwar in einem solchen Maß, dass sie eine positive Nettoauswirkung auf den Erhaltungszustand der Wolfspopulation hätte.“ (Generalanwalt Saugmandsgaard Øe, Schlussanträge v. 8.5.2019C-674/17, ECLI:EU:C:2019:394, Rdnr. 60 u. 62). 
stehen einer illegalen Aktivität wie der Wilderei oder die Schwierigkeiten, denen bei der Umsetzung der Kontrolle dieser Aktivität begegnet wird, könne nicht genügen, um einen Mitgliedstaat von seiner Pflicht zu entbinden, den Schutz der gemäß der FFH-Richtlinie geschützten Arten zu gewährleisten. ${ }^{33}$ Anderenfalls würde die Vorgabe verfehlt, ein strenges, ,praktisch wirksames“ Schutzsystem zu schaffen, das imstande ist, tatsächlich absichtliche Formen des Fangs oder der Tötung von aus der Natur entnommenen Exemplaren der in Anhang IV lit. a) FFH-Richtlinie genannten Tierarten zu verhindern. ${ }^{34}$ Aus diesem Grund dürfe eine Ausnahme auch nur dann genehmigt werden, wenn das mit dieser Ausnahme verfolgte Ziel - als weitere Ausprägung des allgemeinen Grundsatzes der Verhältnismäßigkeit ${ }^{35}$ - nicht durch eine anderweitige zufriedenstellende Lösung erreicht werden kann, wobei den betreffenden Mitgliedstaat eine Begründungspflicht treffe. Dies zugrunde gelegt und vorbehaltlich der vom vorlegenden Gericht vorzunehmenden Überprüfung enthalte die Vorlageentscheidung, so der EuGH, keine Anhaltspunkte dafür, dass die Behörde nachgewiesen hätte, dass das einzige Mittel zur Erreichung des Ziels, das zur Begründung von Ausnahmen zwecks Bestandspflege geltend gemacht wird, darin besteht, ein gewisses $\mathrm{Maß}$ an bestandspflegender Jagd auf den Wolf gemäß Art. 16 Abs. 1 lit. e) FFH-Richtlinie zuzulassen. ${ }^{36}$

Den vorläufigen Schlusspunkt dieser Entwicklung markiert das Urteil des EuGH vom 11.6.2020, welches das generelle Ausnahmeregime des Art. 16 Abs. 1 FFH-Richtlinie anhand eines Wolfsfangs in der Republik Rumänien in den Blick nimmt. Darin bekräftigt der Gerichtshof einmal mehr, dass die Mitgliedstaaten nicht nur verpflichtet seien, einen „vollständigen gesetzlichen Rahmen“ zu schaffen, sondern auch konkrete besondere Schutzmaßnahmen in ,kohärenter und koordinierter" Form durchzuführen, die es erlauben, absichtliche Formen des Fangs oder der Tötung von aus der Natur entnommenen Exemplaren geschützter Tierarten tatsächlich zu verhindern. ${ }^{37}$ Dabei sei der Ausdruck ,aus der Natur“ schon dem Normtext nach nicht mit dem Begriff des „natürlichen Lebensraums“ i.S.d. Art. 3 bis Art. 6 FFH-Richtlinie identisch. Der durch Art. 12 Abs. 1 FFH-Richtlinie gewährte (Arten-)Schutz kenne seines Ziels wegen gerade keine Abgrenzungen oder Grenzen und lasse daher nicht den Schluss zu, dass ein wildlebendes Exemplar einer geschützten Tierart, das sich in der Nähe oder innerhalb von menschlichen Siedlungsgebieten befindet, das solche Gebiete durchquert oder sich von Ressourcen ernährt, die der Mensch erzeugt, ein Tier wäre, das sein ,natürliches Verbreitungsgebiet" verlassen hat. ${ }^{38}$ Dies hat zur Folge, dass das strenge Ausnahmeregime des Art. 16 Abs. 1 FFH-Richtlinie, wonach alle Formen des absichtlichen Fangs von Exemplaren geschützter Tierarten verboten sind, wenn die zuständige nationale Behörde keine Ausnahme auf seiner Grundlage gewährt hat, ${ }^{39}$ auch für den Wolf gilt - einer Tierart, die von Natur aus große Lebensräume beansprucht und in zahlreichen Regionen der Union in vom Menschen beanspruchten Gebieten in unmittelbarer Nähe menschlicher Niederlassungen lebt.

\subsection{Zur deutschen Regelung im Einzelnen}

Im nationalen Recht dienen $\$ \int 44,45$ BNatSchG der Umsetzung von Art. $12 \mathrm{ff}$. FFH-Richtlinie. Von den in $\$ 44$ Abs. 1 und 2 BNatSchG geregelten Verboten kann nur in den gesetzlich vorgesehenen Fällen eine Ausnahme gewährt werden. $₫ 45$ Abs. $7 \mathrm{BNatSchG}$ ist dabei an den sich aus Art. 16 Abs. 1 FFH-Richtlinie (und der entsprechenden Ausnahmebestimmung des Art. 9 Abs. 1 VogelschutzRichtlinie ${ }^{40}$ ) ergebenden Anforderungen an Ausnahmegenehmigungen im Einzelfall zu messen.

Zweifel an der Vereinbarkeit des neuen $\$ 45$ a Abs. 2 BNatSchG mit artenschutzrechtlichen Vorgaben wurden bereits im Verlauf des Gesetzgebungsverfahrens von verschiedener Seite geäußert. ${ }^{41}$ Problematisch erscheint zum einen, ob $\$ 45$ a Abs. 2 BNatSchG unzulässigerweise auf die Geeignetheit der Entnahmeentscheidung zur Erreichung der in $\$ 45$ Abs. 7 S. 1 Nr. 1 bzw. Nr. 4 genannten Ziele verzichtet (dazu unter 3.3.1). Des Weiteren soll die Möglichkeit der Entnahme eines kompletten Rudels (s. u. 3.3.2) ebenso hinterfragt werden wie fehlende Regelungen zur Koordinierung von Maßnahmen des Wolfsmanagements (3.3.3). Schließlich bestehen Zweifel an der hinreichenden Bestimmtheit der neuen Regelung (3.3.4).

\subsubsection{Geeignetheit der Entnahmeentscheidung?}

Steht sicher fest, dass ein bestimmtes Tier einen ernsten wirtschaftlichen Schaden verursachen wird oder eine Gefahr für die menschliche Gesundheit darstellt, so ist die Entnahme eben dieses Tieres geeignet, den drohenden Schaden abzuwenden. Da Ausnahmen von den geltenden artenschutzrechtlichen Verboten allein zur Erreichung bestimmter Ziele genehmigt werden dürfen, ist im Rahmen der Prüfung des $₫ 45$ Abs. 7 S. 1 BNatSchG daher jeweils die Identifizierung des konkret schadensverursachenden Tieres erforderlich. Zwar hält der Gesetzgeber auch nach Änderung der Bestimmungen formal am Kriterium der Geeignetheit fest, ${ }^{42}$ doch nimmt $₫ 45$ a Abs. 2 nun bewusst Abstriche in Kauf. Vor dem Hintergrund praktischer Schwierigkeiten ${ }^{43}$ soll der Abschuss einzelner Mitglieder des Wolfsrudels bei fehlender Möglichkeit der konkreten Zuordnung von Nutztierrissen zu einem bestimmten Wolf eines Rudels schon zulässig sein, wenn er in engem räumlichem und zeitlichem Zusammenhang zu den aufgetretenen Rissereignissen erfolgt. Ob damit künftige Schäden tatsächlich vermieden werden, ist allerdings in diesem Fall gerade zweifelhaft. Tatsächlich verlangt zumindest die Gesetzesbegründung nicht einmal, dass die Schäden sicher dem betroffenen Wolfsrudel zugeordnet wurden; es müsse nur „mit hoher Wahrscheinlichkeit ausgeschlossen werden, dass es sich etwa um einen Riss durch Hunde oder um eine bloße Nachnutzung durch den Wolf handelt". ${ }^{44}$

33) $\mathrm{EuGH,} \mathrm{Urt.} \mathrm{v.} \mathrm{10.10.2019} \mathrm{-} \mathrm{C-674/17,} \mathrm{NuR} \mathrm{2019,} 756$ Rdnr. 48.

34) Vgl. EuGH, Urt. v. 10.10.2019 - C-674/17, NuR 2019, 756, Rdnr. 27 u. 36.

35) Generalanwalt Saugmandsgaard Øe, Schlussanträge v. 8.5.2019 C-674/17, ECLI:EU:C:2019:394, Rdnr. 47 m.w.N.

36) EuGH, Urt. v. 10.10.2019 - C-674/17, NuR 2019, 756, Rdnr. 52.

37) EuGH, Urt. v. 11. 6.2020 - C-88/19, NuR 2020, 468, Rdnr. 23.

38) EuGH, Urt. v. 11.6.2020 - C-88/19, NuR 2020, 468, Rdnr. 39. Ebenso Generalanwältin Kokott, Schlussanträge v. 13.2.2020 C-88/19, ECLI:EU:C:2020:93, Rdnr. 35 f.u. 49.

39) $\mathrm{EuGH}$, Urt. v. 11. 6.2020 - C-88/19, NuR 2020, 468, Rdnr. 56 u. 62. Ausführlich zu den Ausnahmen nach Art. 16 Abs. 1 FFH-Richtlinie: Generalanwältin Kokott, Schlussanträge v. 13.2.2020 - C-88/19, ECLI:EU:C:2020:93, Rdnr. $56 \mathrm{ff}$.

40) S. bereits unter 1.

41) S. Patt, Stellungnahme zum Entwurf eines Zweiten Gesetzes zur Änderung des BNatSchG, Ausschussdrucksache 19(16)308-A, S. 2, 5; Gellermann, Stellungnahme zum Entwurf eines Zweiten Gesetzes zur Änderung des BNatSchG, Ausschussdrucksache 19(16)308-C, S. 4f.; Kremer, Stellungnahme zum Entwurf eines Zweiten Gesetzes zur Änderung des BNatSchG, Ausschussdrucksache 19(16)308-D, S. 5 ff.; vgl. auch Beschlussempfehlung und Bericht des Ausschusses für Umwelt, Naturschutz und nukleare Sicherheit, BT-Drs. 19/16148, S. 8f.; Plenar-Prot. des Deutschen Bundestages 19/137, 17160 D; sowie Empfehlung der Ausschüsse, BR-Drs. 26/1/20, S. 1 f.; a. A. die Begründung des Gesetzentwurfs der Bundesregierung, BT-Drs. 19/10899, S. 7.

42) So der Gesetzentwurf der Bundesregierung, BT-Drs. 19/10899, S. 10.

43) Vgl. wiederum den Gesetzentwurf der Bundesregierung, BTDrs. 19/10899, S. 10

44) Gesetzentwurf der Bundesregierung, BT-Drs. 19/10899, S. 10. 
Auch ob die praktischen Probleme im Umgang mit Ausnahmegenehmigungen zur Entnahme von Wölfen damit der Vergangenheit angehören, ist ungewiss. Denn Probleme bereitet in der Praxis nicht lediglich die konkrete Zuordnung von Rissereignissen, sondern auch die Inanspruchnahme bereits erteilter Entnahmeentscheidungen. ${ }^{45}$ Nicht zuletzt vor diesem Hintergrund wird in der Rechtsprechung - gestützt auf die Gesetzesbegründung - bereits eine erweiternde Auslegung erwogen. ${ }^{46}$ Gerade eine über den Wortlaut noch hinausgehende Auslegung würde allerdings die Bedenken mit Blick auf die Bestimmtheit der Ausnahmeregelung ${ }^{47}$ noch verstärken.

Im Lichte der dargestellten unionsrechtlichen Anforderungen $^{48}$ ist $\$ 45$ a Abs. 2 S. 1 (ggf. i. V.m.S. 3) BNatSchG jedenfalls restriktiv auszulegen und anzuwenden. Zwar erkennt der EuGH praktische Schwierigkeiten der konkreten Zuordnung eingetretener Rissereignisse $\mathrm{zu}$ einem bestimmten Einzeltier durchaus an, ${ }^{49}$ am Erfordernis eines Nachweises der Geeignetheit der Entnahme einzelner Tiere zur Verhütung ernster Schäden i.S.d. Art. 16 Abs. 1 lit. b) FFH-RL hat der Gerichtshof jedoch festgehalten ${ }^{50}$ und darüber hinaus jüngst betont, dass die Begründung der Geeignetheit auf der Grundlage streng wissenschaftlicher Erkenntnisse zu untermauern sei. ${ }^{51}$

Daher wird die Zulassung einer Entnahme eines Wolfs auch in Anwendung des neuen $₫ 45$ a Abs. 2 S. 1 BNatSchG konkrete Ausführungen zur Geeignetheit der Maßnahme erfordern. ${ }^{52}$ Insbesondere kann nicht von vornherein auf den Versuch der Identifizierung des schadensverursachenden Tieres verzichtet werden; alle zumutbaren Mittel der konkreten Zuordnung der Schäden sind auszuschöpfen. ${ }^{53}$ Dabei muss jedenfalls feststehen, dass aufgetretene Rissereignisse überhaupt durch einen Wolf verursacht wurden; auf diese Zuordnung verzichtet auch $\$ 45$ a Abs. 2 S. 1 BNatSchG nicht.

Kann die Geeignetheit nicht hinreichend auf Grundlage wissenschaftlicher Erkenntnisse dargelegt werden, so ist eine Entnahmeentscheidung auch nach $\int 45$ a Abs. 2 S. 1 BNatSchG unzulässig. Dass Möglichkeiten einer weiteren Konkretisierung durchaus möglich sind, zeigen erste landesrechtliche Regelungen, auf die noch zurückzukommen sein wird. ${ }^{54}$

\subsubsection{Entnahme des kompletten Wolfsrudels}

Eng verknüpft mit der Frage, ob eine auf Grundlage des $\$ 45$ a Abs. 2 S. 1 BNatSchG zugelassene Entnahme von Wölfen überhaupt geeignet ist, die anerkannten Ziele zu erreichen, ist die Frage nach der Geeignetheit und generellen Zulässigkeit einer wiederholten Entnahme - möglicherweise bis hin zur Entnahme eines kompletten Rudels. Dabei ist zunächst klarzustellen, dass auch die neu ins Bundesnaturschutzgesetz aufgenommene Regelung keinen Automatismus vorsieht. Vielmehr müssen für jede einzelne Entnahme eines Wolfes die entsprechenden Voraussetzungen erfüllt sein und es stellt sich vor jeder Genehmigung zur Entnahme eines (weiteren) Wolfes erneut die bereits angesprochene Frage der Geeignetheit. Wurde bereits eine Entnahme genehmigt und in der Praxis vollzogen, so muss das entnommene Tier daher auch zunächst daraufhin untersucht werden, ob es sich um den "Schadensverursacher“ handelt. ${ }^{55}$ Darüber hinaus befreit auch $₫ 45$ a Abs. 2 S. 1 BNatSchG die für die Entnahmeentscheidung zuständige Behörde nicht von der Prüfung der weiteren Voraussetzungen nach $\int 45$ Abs. 7 BNatSchG. Dies stellt $\int 45$ a Abs. 2 S. 4 BNatSchG ausdrücklich klar. Danach gilt insbesondere weiterhin das Erfordernis der Prüfung zumutbarer Alternativen vor Erteilung einer Genehmigung zur Entnahme eines (weiteren) Wolfs. ${ }^{56}$ Zudem darf sich der Erhaltungszustand der Populationen der Art Wolf nicht verschlechtern. $\mathrm{Ob}$ aber gerade die Entnahme eines kompletten Rudels tatsächlich ohne negative Auswirkungen auf den Erhaltungszustand der Population bleibt, erscheint zweifelhaft und bedarf in der Praxis zumindest näherer Darlegung.
Mit der Formulierung des $₫ 45$ Abs. 7 S. 2 BNatSchG, der Erhaltungszustand der Populationen dürfe sich nicht verschlechtern, weicht der deutsche Gesetzgeber immerhin schon vom Wortlaut der FFH-Richtlinie ab. So verlangt doch Art. 16 Abs. 1 FFH-Richtlinie, ,daß die Populationen der betroffenen Art [...] in einem günstigen Erhaltungszustand verweilen". Im Einklang mit der Rechtsprechung des $\mathrm{EuGH}^{57}$ sieht das nationale Recht allerdings vor, dass Ausnahmen auch dann in Betracht kommen, wenn für die betreffenden Populationen noch kein günstiger Erhaltungszustand festzustellen ist, solange sich dieser Erhaltungszustand nur nicht weiter verschlechtert und die Herstellung eines günstigen Erhaltungszustands nicht behindert wird. ${ }^{58}$

Vor diesem Hintergrund sind somit vor jeder Zulassung einer (weiteren) Entnahmemaßnahme deren Auswirkungen auf den Erhaltungszustand der betroffenen Population zu beurteilen. Allein die Feststellung eines allgemeinen positiven Trends der Entwicklung der Wolfspopulationen in der Bundesrepublik ${ }^{59}$ vermag diese Prüfung nicht zu ersetzen. ${ }^{60}$ Sollte die betroffene Population einen günstigen Erhaltungszustand erreicht haben, so erübrigt sich diese Pro-

45) Vgl. dazu das in Plenar-Prot. des Deutschen Bundestages 19/137, 17160 D genannte Beispiel.

46) Siehe OVG Lüneburg, Beschl. v. 26.6.2020 - 4 ME 116/20, juris Rdnr. 45.

47) Dazu sogleich noch unter 3.3.4.

48) S. o. unter 3.2, in und bei Fn. 30.

49) EuGH, Urt. v. 14. 6.2007-C-342/05, NuR 2007, 477, Rdnr. 41

50) EuGH, Urt. v. 14. 6.2007-C-342/05, NuR 2007, 477, Rdnr. 42 47.

51) S. dazu EuGH, Urt. v. 10.10.2019 - C-674/17, NuR 2019, 756, Rdnr. 44f.; insofern ebenfalls krit. zur Einführung des $\$ 45 \mathrm{a}$ Abs. 2: Borwieck, ZUR 2020, 50, 52.

52) Dies betont auch OVG Lüneburg, Beschl. v. 26.6.2020 - $4 \mathrm{ME}$ $116 / 20$, juris Rdnr. 47.

53) S. dazu auch Gellermann, Stellungnahme zum Entwurf eines Zweiten Gesetzes zur Änderung des BNatSchG, Ausschussdrucksache 19(16)308-C, S. 5, und Kremer, Stellungnahme zum Entwurf eines Zweiten Gesetzes zur Änderung des BNatSchG, Ausschussdrucksache 19(16)308-D, S. 6.

54) Zur Regelung der Sächsischen Wolfsmanagementverordnung unter 4.

55) S. auch den Gesetzentwurf der Bundesregierung, BT-Drs. 19/ 10899, S. 10.

56) Als zumutbare Alternativen sind insbesondere das Einrichten bzw. Verstärken von Herdenschutzmaßnahmen oder etwa die Durchführung von Vergrämungsmaßnahmen in Betracht zu ziehen; dazu u. a. Köck, NuR 2018, 812, 815 f. Eine Alternativenprüfung hat auch die bereits erwähnte Entscheidung des OVG Lüneburg, Beschl. v. 26.6.2020 - 4 ME 116/20, juris Rdnr. $37 \mathrm{f}$. vorgenommen.

57) EuGH, Urt. v. 14.6.2007 - C-342/05, NuR 2007, 477, Rdnr. 29; EuGH, Urt. v. 10.10.2019 - C-674/17, NuR 2019, 756, Rdnr. 68.

58) S. dazu BVerwG, Beschl. v. 17.4.2010 - 9 B 5.10, NuR 2010, 492 , 1. Leitsatz, Rdnr. 8; Lau, NVwZ 2011, 461, 465 f.; Steeck, NuR 2011, 4, 5 f.; Köck, NuR 2018, 812, 814. Näher dazu Gläß, in: Giesberts/Reinhardt (Hrsg.), BeckOK-Umweltrecht, 54. EL (4/2020), $\$ 45$ BNatSchG Rdnr. 59f.; Lau, in: Frenz/Müggenborg, BNatSchG, 2. Aufl. 2016, $₫ 45$ Rdnr. 25f.; Lütkes, in: Lütkes/Ewer, BNatSchG, 2. Aufl. 2018, \$4 45 Rdnr. 58 ff., jeweils m.w. N.

59) S. etwa die Nachweise bei Rüwe, NdsVBl. 2020, 65, 66, in und bei Fn. 26, sowie S. 69. Auch OVG Lüneburg, Beschl. v. 26.6.2020 - 4 ME 116/20, juris Rdnr. 39 stellt auf die positive Entwicklung der Wolfspopulationen in Deutschland ab.

60) Zur erforderlichen Prognose vgl. Köck, Kurzgutachten zum Entwurf „Bayerischer Aktionsplan Wolf“ des Bayerischen Landesamt für Umwelt, 2018, S. 8f., Stand 29.6.2020, abrufbar unter https://www.umweltstiftung.com/fileadmin/archiv/bayern_wild_historie/Koeck-Kurzgutachten-Wolf.pdf. Vor diesem Hintergrund kritisch zur Entnahme des gesamten Rudels auch Gellermann, Stellungnahme zum Entwurf eines Zweiten Gesetzes zur Änderung des BNatSchG, Ausschussdrucksache 19(16)308-C, S. 5, sowie Rüwe, NdsVBl. 2020, 65, 70. 
gnose auch dann nicht, hält doch Art. 16 FFH-Richtlinie gerade fest, dass eine Ausnahme den günstigen Erhaltungszustand nicht beeinträchtigen dürfe. Hat die Entnahme eines (weiteren) Wolfs eine Verschlechterung des Erhaltungszustands zur Folge, so ist diese Entnahme daher auch nach $\int 45$ a Abs. 2 S. 1 BNatSchG unzulässig.

\subsubsection{Wolfsmanagementplan und -koordinierung}

Wolfsmanagement, verstanden als das umfassende Handeln von Naturschutzbehörden, um durch Prävention, Gefahrenabwehr und Monitoring die Managementmaßnahme der Nutzung von Ausnahmen nach $₫ 45$ Abs. 7 S. 1 BNatSchG soweit wie möglich zu vermeiden und dadurch insgesamt dem Artenschutz zu dienen, ${ }^{61}$ lässt sich als Vorgabe der FFH-Richtlinie begreifen, die ein strenges Schutzsystem für die in ihrem Anhang IV lit. a) genannten Tierarten aufrichtet. Dies lässt sich besonders an der Verpflichtung zum Wolfsmonitoring (Art. 11 FFH-Richtlinie) sowie an der in Art. 16 Abs. 1 FFH-Richtlinie niedergelegten Bedingung ablesen, dass die Populationen der betroffenen Art in ihrem natürlichen Verbreitungsgebiet trotz der Ausnahmeregelung ohne Beeinträchtigung in einem günstigen Erhaltungszustand verweilen. Dabei hat sich diese Betrachtung nicht auf das lokale Gebiet oder auf das Hoheitsgebiet des betreffenden Mitgliedstaats zu beschränken, sondern erstreckt sich gegebenenfalls auf die betreffende biogeografische Region - hier insbesondere die sog. Mitteleuropäische Flachlandpopulation -, wenn sich die Grenzen dieses Mitgliedstaats mit mehreren biogeografischen Regionen überschneiden oder wenn das natürliche Verbreitungsgebiet der Art dies erfordert. ${ }^{62} \mathrm{Um}$ diesen Anforderungen gerecht $\mathrm{zu}$ werden, sind Managementpläne, wie sie zum Beispiel $\$ 2$ Sächsische Wolfsmanagementverordnung vorsieht, der sogar das (grenzüberschreitende) Zusammenwirken mit anderen Behörden vorschreibt, eine Möglichkeit des Nachweises ${ }^{63}$, ohne dass eine bundesgesetzliche Verankerung oder ihr Erlass im Einzelfall unionsrechtlich zwingend vorgegeben wären. ${ }^{64}$ Ebenso wenig bedarf es einer spezifischen Normierung des Erfordernisses der Koordinierung und Abstimmung des Wolfsmanagements zwischen den beteiligten Ländern in $\ 45 \mathrm{a}$ BNatSchG ${ }^{65}$ Für die Bundesländer ergibt sich diese Pflicht ohnehin aus dem Grundsatz der Bundestreue, während sie sich für die Mitgliedstaaten der Europäischen Union aus Art. 4 Abs. 3 EUV herleiten lässt. ${ }^{66}$ Wolfsmanagement ist im Übrigen eine Frage des Normvollzugs, der in Deutschland dezentral erfolgt (Art. 83, 84 GG). Hält man sich dies und den Umstand vor Augen, dass sich die Unterscheidung zwischen Legislative und Exekutive auch in der Rechtsprechung des EuGH manifestiert, wird man diese am ehesten so verstehen dürfen, dass sich die bereits erwähnte Verpflichtung zum „Erlass kohärenter und koordinierter vorbeugender Maßnahmen“ zuvörderst an die nationalen Behörden richtet. ${ }^{67}$

\subsubsection{Bestimmtheit der Sonderregelung}

Dagegen ist die dort ebenfalls ausgesprochene Verpflichtung, einen ,,vollständigen gesetzlichen Rahmen“ zu schaffen, zweifellos an den mitgliedstaatlichen Gesetzgeber adressiert. Dem wird er nicht schon dadurch gerecht, dass er einen Hinweis in $\$ 45$ a Abs. 2 S. 4 BNatSchG aufnimmt, der auf die Notwendigkeit der Prüfung der weiteren Ausnahmevoraussetzungen in $\$ 45$ Abs. 7 S. 2 und 3 BNatSchG aufmerksam macht ${ }^{68}$. Im Gegenteil wirft gerade diese Regelungstechnik die Frage auf, ob der deutsche Gesetzgeber die Ausnahme „klar, genau und fundiert“ festgelegt hat. Diese Vorgabe gilt sowohl für die behördliche Einzelfallentscheidung zur Erteilung einer Ausnahme als auch für den Gesetzgeber selbst ${ }^{69}$, da in beiden Fällen die praktische Wirksamkeit des strengen Schutzsystems der FFH-Richtlinie auf dem Spiel steht. Speziell für derartige Ausnahmeregelungen gelten also gesteigerte Anforderungen an die Normenbestimmtheit und -klarheit. In der Rechtspre- chung des EuGH klingt das dann so: „Der Genauigkeit der Umsetzung kommt allerdings besondere Bedeutung $\mathrm{zu}$ in einem Fall wie dem vorliegenden, in dem die Verwaltung des gemeinsamen Erbes den Mitgliedstaaten für ihr jeweiliges Hoheitsgebiet anvertraut ist." ${ }^{30}$ Weiter merkt der EuGH an, dass die richtlinienkonforme Ausführung der Vorschriften durch nationale Behörden grundsätzlich nicht die Klarheit und Genauigkeit aufweisen könne, um den Anforderungen der Rechtssicherheit zu genügen. ${ }^{71} \mathrm{Da}-$ bei wäre es - isoliert betrachtet - vielleicht noch hinnehmbar, dass $₫ 45 \mathrm{a}$ Abs. 2 S. 4 BNatSchG als ein Einfallstor für die unionsrechtskonforme Auslegung fungiert und insoweit maßgeblich dazu beiträgt, der (sukzessiv erteilten) Genehmigung zur Entnahme einzelner nicht schadensverursachender Wölfe bis hin zu einem kompletten Rudel Grenzen zu setzen ${ }^{72}$. Doch hat es damit nach dem oben Gesagten nicht sein Bewenden. Es ist nicht nur der Umstand, dass $₫ 45$ Abs. 7 S. 1 Nr. 1 BNatSchG hinsichtlich der erfassten Schadenspositionen textlich von Art. 16 Abs. 1 lit. b) FFH-Richtlinie abweicht und insofern der unionsrechtskonformen Auslegung bedarf: ${ }^{73}$ vor allem fehlt es - sowohl in $\$ 45$ a Abs. 2 BNatSchG als auch in der dazugehörigen Gesetzesbegründung - an einer Angabe der erforderlichen Anzahl von Rissereignissen sowie an konkreten Kriterien für die Bemessung des ,engen räumlichen und zeitlichen Zusammenhangs"74. Hinzu kommt, dass man $\$ 45$ a Abs. 2 S. 1 BNatSchG trotz des an sich anderslautenden Wortlauts dahingehend wird verstehen müssen, dass eine Tötung erst dann in Betracht kommt, wenn die Zuordnung zu einem bestimmten Wolf eines Rudels konkret und mit einem gewissen Aufwand versucht worden ist, da die Zuordnung

61) Vgl. \$1 Nr. 4 Sächsische Wolfsmanagementverordnung - SächsWolfMVO (Wolfsmanagementverordnung des Sächsischen Staatsministeriums für Umwelt und Landwirtschaft v. 15. 5. 2019, SächsGVBl. S. 332). Zum Begriff des Wolfsmanagements, den Zuständigkeiten und zu seinem Inhalt, vgl. nur Köck/Kuchta, NuR 2017, 509, $512 \mathrm{ff}$.

62) So EuGH, Urt. v. 10.10.2019 - C-674/17, NuR 2019, 756, Rdnr. 58. Für ein Abstellen auf die biogeografische Region unter Einbeziehung von Teil-Populationen in den Nachbarstaaten bereits Köck, NuR 2018, 812, $815 \mathrm{f}$.

63) Die Kommission hält den Erlass von Managementplänen für ,die beste Möglichkeit [...], die Einhaltung der strengen Anforderungen von Artikel 16 [FFH-Richtlinie] nachzuweisen." (Leitfaden der Kommission, S. 64, Nr. 33, Stand 29.6.2020, abrufbar unter https://ec.europa.eu/environment/nature/conservation/species/ guidance/pdf/guidance_de.pdf).

64) Bei EuGH, Urt. v. 10.10.2019 - C-674/17, NuR 2019, 756 findet sich weder eine Erwähnung von Managementplänen noch eine Bezugnahme auf die entsprechenden Ausführungen des Generalanwalts (s. Generalanwalt Saugmandsgaard Øe, Schlussanträge v. 8. 5. 2019 - C-674/17, ECLI:EU:C:2019:394, Rdnr. 94f.).

65) A. A. Borwieck, ZUR 2020, 50, 53.

66) Köck, NuR 2018, 812, 816.

67) Vgl. EuGH, Urt. v. 10.10.2019 - C-674/17, NuR 2019, 756, Rdnr. 27 (Hervorhebung nur hier).

68) Vgl. BT-Drs. 19/16148, S. 10.

69) (Nur) auf die behördliche Einzelfallentscheidung bezogen EuGH, Urt. v. 10.10.2019 - C-674/17, NuR 2019, 756, Rdnr. 41, der allerdings Art. 16 Abs. 1 FFH-Richtlinie selbst eine ,genaue und abschließende" Festlegung attestiert (ebd., Rdnr. 30).

70) EuGH, Urt. v. 8.7.1987 - 247/85, ECLI:EU:C:1987:339, Rdnr. 9; ähnlich EuGH, Urt. v. 27.4.1988 - 252/85, ECLI:EU:C:1988:202, Rdnr. 5; Urt. v. 10.1.2006 - C-98/03, NuR 2006, 166, Rdnr. 59.

71) EuGH, Urt. v. 15.3.2012 - C-46/11, ZUR 2013, 489, 491.

72) S. oben 3.3.1 und 3.3.2.

73) Vgl. Borwieck, ZUR 2020, 50, 52.

74) So auch Gellermann, Stellungnahme zum Entwurf eines Zweiten Gesetzes zur Änderung des BNatSchG, Ausschussdrucksache 19(16)308-C, S. 4; Kremer, Stellungnahme zum Entwurf eines Zweiten Gesetzes zur Änderung des BNatSchG, Ausschussdrucksache 19(16)308-D, S. 5 f. 
eines Schadens zu einem bestimmten Tier im Sinne des Schutzregimes der FFH-Richtlinie grundsätzlich als mildere Maßnahme anzusehen ist.

\section{Konkretisierungen im sächsischen Landesrecht}

Wie dargelegt, bestehen mit Blick auf die neu erlassene bundesrechtliche Regelung in verschiedener Hinsicht Bedenken, die jedenfalls eine restriktive Auslegung und Anwendung des $\$ 45$ a Abs. 2 BNatSchG erforderlich machen. Die bundesrechtliche Regelung ersetzt zudem nicht die Umsetzung auf landesrechtlicher Ebene, die notwendigen Entscheidungen im Einzelfall, die durch die nach Landesrecht zuständigen Behörden zu treffen sind. Vor diesem Hintergrund lohnt ein Blick auf Regelungen, die auf landesrechtlicher Ebene bereits zuvor getroffen worden waren - ebenfalls mit dem Ziel, den Vollzug der artenschutzrechtlichen Bestimmungen im Umgang mit dem Wolf einfacher und rechtssicher auszugestalten.

\subsection{Regelungsgegenstand der Sächsischen Wolfsmanagementverordnung}

Neben dem Land Brandenburg hat der Freistaat Sachsen gestützt auf die Ermächtigung des $\$ 45$ Abs. 7 S. 4 BNatSchG eine sog. Wolfsverordnung erlassen. Danach können die Landesregierungen Ausnahmen auch allgemein durch Rechtsverordnung zulassen. Dabei lassen sich zwar gewisse Parallelen zur bundesrechtlichen Regelung des $\$ 45$ a Abs. 2 BNatSchG feststellen, allerdings trifft der Freistaat Sachsen zum Teil deutlich konkretere Regelungen, die unter Umständen auch als Anhaltspunkt für die erforderliche restriktive Auslegung des $\$ 45 \mathrm{a}$ Abs. $2 \mathrm{BNatSchG}$ herangezogen werden können.

Die sächsische Wolfsmanagementverordnung enthält neben Bestimmungen über den vom LfULG aufzustellenden Managementplan Wolf ( $\$ 2$ SächsWolfMVO) sowie Vorgaben zum Monitoring und zu Rissbegutachtung ( $\$ 3$ SächsWolfMVO) insbesondere Regelungen über die Vergrämung und Entnahme von Wölfen in verschiedenen Fällen ( $\iint 5 \mathrm{ff}$. SächsWolfMVO). Eine Entnahme von Wölfen wird durch die Verordnung als Ausnahme zugelassen,

- zur Vermeidung erheblicher wirtschaftlicher Schäden durch Risse von landwirtschaftlich gehaltenen Schafen und Ziegen (\$ 6 SächsWolfMVO);

- im Fall von Wolfshybriden ( $\$ 7$ SächsWolfMVO);

- zum Schutz der menschlichen Gesundheit gegenüber sich unprovoziert aggressiv verhaltenden Wölfen ( $\$ 8$ SächsWolfMVO);

- im Fall schwer verletzter oder erkrankter Wölfe (\$ 11 SächsWolfMVO);

- sowie aus sonstigen zwingenden Gründen des überwiegenden öffentlichen Interesses ( $\$ 9$ SächsWolfMVO).$^{75}$

\subsection{Entnahme von Wölfen nach sächsischem Landesrecht}

Mit den Regelungen der $\int S 6-8$ weist die sächsische Verordnung Parallelen zur bundesrechtlichen Regelung des $\$ 45 \mathrm{a}$ BNatSchG auf. Im Unterschied zu $₫ 45 \mathrm{a}$ Abs. 2 S. 2 stellt $₫ 6$ Abs. 1 S. 1 Nr. 1 SächsWolfMVO klar auf den Schutz erheblicher betriebswirtschaftlicher Werte ab, indem eben solche zwingend im Gebiet des den Schaden verursachenden Wolfs vorhanden sein müssen. Zwar wird auch die Überwindung von Maßnahmen zum Schutz nicht landwirtschaftlich gehaltener Schafe oder Ziegen berücksichtigt, allerdings lediglich mit Blick auf die Frage, ob der zu entnehmende Wolf Schutzmaßnahmen wiederholt ${ }^{76}$ überwunden hat. Die Verordnung bestimmt im Übrigen nicht lediglich die Anforderungen an derartige Schutzmaßnahmen in der Anlage näher, sondern legt auch fest, dass diese - ordnungsgemäß errichteten und funktionstüchtig betriebenen - Schutzmaßnahmen zweimal innerhalb von zwei Wochen (\$6 Abs. 1 S. 1 Nr. 2 SächsWolfMVO) überwunden werden müssen bzw. generell zweimal, soweit es um den Schutz von Gehegewild geht ( $\$ 6$ Abs. 3 SächsWolfMVO).

Zwar sieht auch $\$ 6$ Abs. 3 SächsWolfMVO die Möglichkeit einer Entnahme von Wölfen vor, wenn der konkret schadensverursachende Wolf nicht sicher identifiziert werden kann, die Entnahme in einem solchen Fall setzt allerdings voraus, dass sich die Schadensorte in einem Rudelterritorium befinden und sich der zu entnehmende Wolf erneut - nach vorheriger Überwindung von Schutzmaßnahmen, wie eben dargestellt - der Schafs-, Ziegen- oder Gehegewildhaltung nähert. Im Interesse des Tierschutzes sieht das Landesrecht in $\$ 6$ Abs. 4 S. 2 SächsWolfMVO zudem eine Begrenzung der Entnahmemöglichkeit von Elterntieren vor, wenn kein Elterntier zur Aufzucht vorhandener Welpen verbleiben würde. Die Entnahme ist nach \10 Abs. 2 Nr. 1 SächsWolfMVO zu dokumentieren und in zeitlicher und räumlicher Hinsicht zu begrenzen.

In vergleichbarer Weise gibt das sächsische Recht auch die Voraussetzungen konkreter vor, unter denen eine Entnahme von Wölfen zum Schutz der menschlichen Gesundheit in Betracht kommt. Eine Ausnahme zu diesem Zweck ist gemäß $₫ 8$ Abs. 1 S. 1 SächsWolfMVO nur zugelassen, wenn

- ein Wolf einen Menschen verletzt, ihn verfolgt oder sich ihm gegenüber in sonstiger Weise unprovoziert aggressiv gezeigt hat,

- sich ein Wolf einem Menschen außerhalb von Gebäuden und Fahrzeugen auf unter 30 Meter nähert, den Abstand zu diesem Menschen duldet und eine Vergrämung erfolglos geblieben ist,

- sich ein Wolf zu Wohnzwecken genutzten Gebäuden, insbesondere bei der Futtersuche wiederholt nähert, eine Vergrämung erfolglos geblieben ist und sich durch die örtlichen Gegebenheiten die Gefahr für eine Annäherung an Menschen auf unter 30 Meter deutlich erhöht oder

- ein Wolf wiederholt Haustiere, insbesondere Hunde im umfriedeten Bereich von Wohngrundstücken tötet, eine Vergrämung erfolglos geblieben ist und sich dadurch die Gefahr für Menschen deutlich erhöht. Ebenso wie im Fall einer Entnahme zum Schutz von Weidetieren müssen die Voraussetzungen hinreichend belegt sein (s. JS6 Abs. 1 S. 3, 8 Abs. 1S. 2 SächsWolfMVO).

Mit Blick auf die europarechtlichen Vorgaben sollen insbesondere die folgenden Punkte der sächsischen Regelung hervorgehoben werden: Das Erfordernis von Belegen und einer Dokumentation trägt den Anforderungen an die wissenschaftliche Begründung der Geeignetheit der Entnahmeentscheidung Rechnung. Die Entnahme von Wölfen ohne konkrete Identifizierung des schadensverursachenden Tieres kommt nur nachrangig, nach $\$ 6$ Abs. 3 der Verordnung in Betracht. ${ }^{77}$ Zugleich wird die Möglichkeit, einen nicht konkret als Schadensverursacher identifizierten Wolf aus der Natur zu entnehmen, hier - anders als auf Grund-

75) Die Auseinandersetzung mit der SächsWolfMVO beschränkt sich hier ausdrücklich auf die Entnahmeregelungen, die Parallelen zur bundesrechtlichen Bestimmung des $₫ 45$ a Abs. 2 BNatSchG aufweisen. Andere rechtliche Fragen bleiben vorliegend außer Betracht.

76) Ergänzend sei darauf hingewiesen, dass auch $\$ 4$ Abs. 2 der Verordnung über die Zulassung von Ausnahmen von den Schutzvorschriften für den Wolf des Landes Brandenburg (Brandenburgische Wolfsverordnung - BbgWolfV) v. 26.1.2018, GVB1. II, Nr. 8 vor einer Entnahme das wiederholte - mindestens zweimalige Überwinden von Schutzmaßnahmen durch den Wolf verlangt.

77) Vgl. die Begründung des Verordnungsentwurfs der Staatsregierung, Stand 16.7.2020, abrufbar unter https://www.wolf.sachsen.de/sachsische-wolfsmanagementverordnung-4043.html, S. 11 
lage des $\ 45$ a Abs. 2 S. 1 BNatSchG - durch zusätzliche Voraussetzungen beschränkt. Ein beliebiger Abschuss von Wölfen eines Rudels kommt nicht in Betracht, vielmehr kann lediglich ein Wolf entnommen werden, der sich (erneut) Weidetieren innerhalb des Rudelterritoriums nähert und damit immerhin Anhaltspunkte liefert, die die fachliche Einschätzung rechtfertigen könnten, dass es sich dabei um den konkret schadensverursachenden Wolf handelt. ${ }^{78}$ Dass eine Entnahme von Wölfen lediglich in Fällen in Betracht kommt, in denen Vergrämungsmaßnahmen dem Schutzzweck nicht hinreichend Rechnung tragen können, trägt zur Einhaltung der europarechtlichen Anforderungen bei, müssen doch auf diese Weise zumutbare Alternativen ausgeschlossen sein.

\section{Schlussbemerkung}

Die erwähnten Ansätze im Landesrecht zeigen, dass Konkretisierungen auch im Bereich artenschutzrechtlicher Ausnahmeregelungen, die praxisrelevante Probleme adressieren sollen, durchaus möglich sind. Die durch $₫ 45$ a Abs. 2 BNatSchG verfolgte Zielsetzung, mehr Rechtssicherheit für die Anwendung der artenschutzrechtlichen Ausnahmebestimmungen im Umgang mit dem Wolf zu schaffen, wurde allerdings verfehlt. Nicht nur bleibt die Regelung dafür in verschiedener Hinsicht zu unbestimmt, insbesondere weckt sie Zweifel an der Vereinbarkeit mit europarechtlichen Anforderungen. Allenfalls im Fall einer restriktiven Auslegung und Anwendung kann $₫ 45 a$ Abs. 2 BNatSchG noch als europarechtskonform angesehen werden. Dabei ist zu erwarten, dass letztlich erst der EuGH für die notwendige - und mit der Regelung ursprünglich angestrebte Rechtssicherheit - sorgen wird, sollte ihm die Bestimmung vorgelegt werden. Bis dahin bleibt es Aufgabe der zuständigen Behörden und nationalen Gerichte, den Vorgaben der FFH-Richtlinie bei Anwendung des $\$ 45 \mathrm{a}$ Abs. 2 BNatSchG Rechnung zu tragen. Ob auf diese Weise Schäden in Form von durch Wölfe verursachten Rissereignissen besser vermieden werden können, bleibt fraglich.

Open Access. Dieser Artikel wird unter der Creative Commons Namensnennung 4.0 International Lizenz veröffentlicht, welche die Nutzung, Vervielfältigung, Bearbeitung, Verbreitung und Wiedergabe in jeglichem Medium und Format erlaubt, sofern Sie den/die ursprünglichen Autor(en) und die Quelle ordnungsgemäß nennen, einen Link zur Creative Commons Lizenz beifügen und angeben, ob Änderungen vorgenommen wurden.

Die in diesem Artikel enthaltenen Bilder und sonstiges Drittmaterial unterliegen ebenfalls der genannten Creative Commons Lizenz, sofern sich aus der Abbildungslegende nichts anderes ergibt. Sofern das betreffende Material nicht unter der genannten Creative Commons Lizenz steht und die betreffende Handlung nicht nach gesetzlichen Vorschriften erlaubt ist, ist für die oben aufgeführten Weiterverwendungen des Materials die Einwilligung des jeweiligen Rechteinhabers einzuholen.

Weitere Details zur Lizenz entnehmen Sie bitte der Lizenzinformation auf http://creativecommons.org/licenses/by/4.0/deed.de.

Open Access funding enabled and organized by Projekt DEAL.

78) Siehe Begründung des Verordnungsentwurfs der Staatsregierung, Stand 16.7.2020, abrufbar unter https://www.wolf.sachsen.de/ sachsische-wolfsmanagementverordnung-4043.html, S. $11 \mathrm{f}$.

\title{
Erleichterungen im besonderen Artenschutz
}

\author{
- die Schlussanträge der Generalanwältin Kokott vom 10.9.2020 im Fall Härryda
}

\section{Marcus Lau}

(c) Springer-Verlag GmbH Deutschland, ein Teil von Springer Nature 2021

Mit ihren Schlussanträgen vom 10.9. 2020 zeigt Generalanwältin Kokott Wege auf, die berechtigten Belange modernen menschlichen Lebens mit dem Schutz seltener und besonders gefährdeter Arten in Einklang zu bringen. Nachdem in den zurückliegenden Jahren und Jahrzehnten das besondere Artenschutzrecht auf europäische Initiative hin, insbesondere durch die Auslegung der diesbezüglichen Bestimmungen in der Vogelschutz- und FFH-Richtlinie durch den $\mathrm{EuGH}$, immer strenger geworden ist, bahnt sich nun möglicherweise eine neue Akzentuierung an. Auch wenn das Urteil des EuGH in dem Fall noch nicht vorliegt, lohnt bereits jetzt ein genauerer Blick auf die Schlussanträge.

\section{Einleitung}

Neben seinen lange Zeit das besondere Artenschutzrecht dominierenden Besitz- und Vermarktungsverboten enthalten die Bestimmungen des Art. $5 \mathrm{~V}-S G^{1}$ und 12 FFH-RL ${ }^{2}$ auch eine Reihe von Zugriffsverboten. Diese sind ebenso wie die Besitz- und Vermarktungsverbote grundsätzlich individuenbezogen ausgestaltet. In Deutschland ist man lange

Dr. Marcus Lau, Rechtsanwalt und Fachanwalt für Verwaltungsrecht, RA Füsser und Kollegen, Leipzig, Deutschland
Zeit davon ausgegangen, dass sich diese Zugriffsverbote auf Handlungen beschränken, deren Zweck der Zugriff auf Exemplare besonders geschützter Arten ist. Seit dem ersten Caretta-Urteil des EuGH im Jahr 2002 war jedoch absehbar, dass sich diese Sichtweise nicht weiter aufrechterhalten lassen wird. ${ }^{3}$ Spätestens mit der Beanstandung des durchweg auf ein absichtliches Handeln abstellenden $₫ 43$ Abs. 4 BNatSchG 2002 durch den EuGH ${ }^{4}$ hatte sich sodann die Einsicht durchgesetzt, dass das besondere Artenschutzrecht über entsprechend intendierte Handlungen hinausreicht und vor allem auch Bauvorhaben betrifft. Seither ist nicht nur der Gesetzgeber mehrmals tätig geworden (2007, 2009 und zuletzt 2017), sondern haben die inzwischen in $\$ 44$

1) Richtlinie 2009/147/EG des Europäischen Parlaments und des Rates vom 30.11.2009 über die Erhaltung der wild lebenden Vogelarten (kodifizierte Fassung), AB1. 2009 L 20, S. 7.

2) Richtlinie 92/43/EWG des Rates vom 21.5.1992 zur Erhaltung der natürlichen Lebensräume sowie der wild lebenden Tiere und Pflanzen, AB1. EU L 206, S. 7.

3) Hierzu Frenz/Lau, in: Frenz/Müggenborg, BKom BNatSchG, 3. Aufl. 2021, Vorb. \$S 44, 45 Rdnr. $11 \mathrm{ff}$.

4) EuGH, Urt. v. 10.1.2006 - C-98/03, ECLI:EU:C:2006:3 (Rdnr. 55), Kommission/Deutschland. 